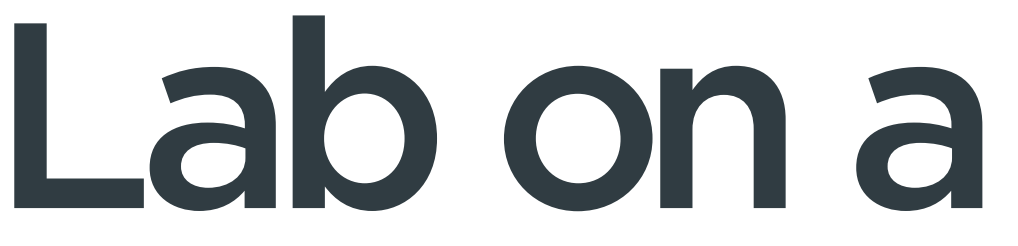

Devices and applications at the micro- and nanoscale

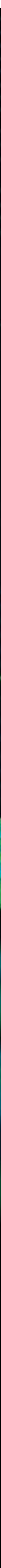

\title{
PAPER
}

Meenesh R. Singh et al. 


\title{
Lab on a Chip
}

Check for updates

Cite this: Lab Chip, 2021, 21, 2333

\section{Advanced continuous-flow microfluidic device for parallel screening of crystal polymorphs, morphology, and kinetics at controlled supersaturation $\dagger$}

\author{
Paria Coliaie, ${ }^{a}$ Manish S. Kelkar, ${ }^{\mathrm{b}}$ Marianne Langston, ${ }^{\mathrm{c}}$ Chengxiang Liu, ${ }^{\mathrm{d}}$ \\ Neda Nazemifard, ${ }^{e}$ Daniel Patience, ${ }^{d}$ Dimitri Skliar, ${ }^{f}$ \\ Nandkishor K. Nere ${ }^{\mathrm{ab}}$ and Meenesh R. Singh (iD*a
}

\begin{abstract}
A flow-controlled microfluidic device for parallel and combinatorial screening of crystalline materials can profoundly impact the discovery and development of active pharmaceutical ingredients and other crystalline materials. While the existing continuous-flow microfluidic devices allow crystals to nucleate under controlled conditions in the channels, their growth consumes solute from the solution leading to variation in the downstream composition. The materials screened under such varying conditions are less reproducible in large-scale synthesis. There exists no continuous-flow microfluidic device that traps and grows crystals under controlled conditions for parallel screening. Here we show a blueprint of such a microfluidic device that has parallel-connected micromixers to trap and grow crystals under multiple conditions simultaneously. The efficacy of a multi-well microfluidic device is demonstrated to screen polymorphs, morphology, and growth rates of L-histidine via antisolvent crystallization at eight different solution conditions, including variation in molar concentration, vol\% of ethanol, and supersaturation. The overall screening time for L-histidine using the multi-well microfluidic device is $\sim 30 \mathrm{~min}$, which is at least eight times shorter than the sequential screening process. The screening results are also compared with the conventional 96-well microtiter device, which significantly overestimates the fraction of stable form as compared to metastable form and shows high uncertainty in measuring growth rates. The multi-well microfluidic device paves the way for next-generation microfluidic devices that are amenable to automation for high-throughput screening of crystalline materials.
\end{abstract}

Received 17th March 2021, Accepted 26th May 2021

DOI: 10.1039/d1lc00218j

rsc.li/loc temperature, and solvent composition. ${ }^{1}$ These devices create cyclonic flow inside the microwell for uniform mixing of a solution while maintaining constant supersaturation. Such devices also overcome the limitations of existing platforms such as continuous flow, well-based, valve-based, and droplet-based microfluidic devices, ${ }^{2}$ which suffer from the depletion of supersaturation. ${ }^{1}$ The continuous-flow microfluidic devices have also evolved to study material synthesis and biological processes under flow-controlled steady-state conditions. ${ }^{3,4}$ Some applications of continuousflow microfluidics include online characterization of nanoparticles, ${ }^{5}$ combinatorial screening of nanoparticles, ${ }^{6}$ parallel syntheses of nanoparticles, ${ }^{7}$ perfusion of cell culture, ${ }^{8}$ cell sorting, ${ }^{9}$ and bioassays. ${ }^{4}$ Specific to materials synthesis, most of the continuous-flow microfluidics utilize flow focusing, annular flow or $\mathrm{T} / \mathrm{Y}$ junctions to induce fast mixing followed by crystal nucleation and growth. In such flow configurations, the nucleated crystals move along the streamlines while consuming the solute and depleting the 
supersaturation in the flowing liquid that often leads to undesired morphologic and polymorphic changes. Maintaining constant supersaturation around the crystals in the flowing medium requires diverging crystals away from the streamlines and isolating them. The rotational flow in the cyclone mixer allows crystals to be separated from the streamlines such that the crystals nucleating in the supersaturated solution can be trapped inside the vortex and grow under constant conditions. Such continuous-flow micromixers have been applied previously to screen crystal morphology, polymorphs and kinetics with higher accuracy and reliability as compared to microtiter plates. ${ }^{1}$ However, these micromixers have not been integrated in microfluidic devices yet to conduct parallel or combinatorial screening for high-throughput applications.

The parallel operation of microchannels is necessary to enable high-throughput screening in continuous-flow microfluidic devices. There are two distinct approaches to automate such parallel operations, namely, valve-controlled (active) and hydraulic-network-controlled (passive) approaches. The valve-controlled approach involves pressureactuated valves for automated sampling, dilution, and mixing, which has been recently applied for parallel operation of eight microchannels for combinatorial screening of up to 648 conditions using six different chemicals in a couple of hours. ${ }^{6}$ Such microfluidic devices can be fully automated and digitally controlled using computers. Another approach is a passive control, where the primary solutions containing different chemicals are distributed and mixed in a split-flow pyramidal hydraulic network to create a range of conditions for parallel and combinatorial screening. ${ }^{10}$ Any of these approaches can be applied to distribute chemicals in individual (single-inlet, single-outlet) microchannels in the device. However, the parallel connection and operation of micromixers that have multiple (up to eight) tangential inlets to create cyclonic flow have not been implemented yet. The design and implementation of such parallelly connected multi-inlets micromixers can have a transformative impact on development of robust materials discovery and screening platforms.

The objective of this article is to design, implement, and evaluate a continuous-flow microfluidic device consisting of fully integrated micromixers for parallel screening of crystal morphology, polymorphs, and growth rates crystals. Here we have engineered the previously reported design of multi-inlet, single-well microfluidic device ${ }^{1}$ to develop a multi-well microfluidic device by consolidating the tangential inlets to eliminate geometric restriction for adjacent wells and the time lag between the inlet flow rates. The 3D printed multiwell microfluidic device is first computationally evaluated and experimentally benchmarked against previously reported screening results of ortho-aminobenzoic acid $(o$-ABA $),{ }^{1}$ and then implemented to screen polymorphs, morphology, and growth rates of L-histidine crystals grown by anti-solvent crystallization. The multi-well microfluidic device is also used to measure the solubility of L-histidine.

\section{Theoretical methods}

\subsection{Design of merged-inlet micromixer in multi-well device}

The alternate tangential inlets of the multi-inlet micromixer (Fig. 1A) can be consolidated into the merged-inlet configuration (Fig. 1B) for their arrangement into an array (Fig. 1C) with fewer inlets to enable parallel screening. Without the merging of inlets, an array of eight micromixers consisting of four inlets each will yield a total of 32 inlets requiring more pumps with increased difficulty to operate. Merging the alternate inlets of the micromixer will increase the effectiveness of mixing and decrease the total number of inlets to half. However, the merging of inlets requires a delicate balance of pressure drop between inlets to allow for uniform splitting of flow and mixing in the micromixer. One possible way to merge the alternate (non-neighboring) inlets is to connect them through a Y-junction while keeping them tangential to the micromixer. The Y-junctions for each pair of inlets have vertical offsets to avoid the intersection of inlet channels. Another requirement is to keep the path of both channels that are merged to be identical to maintain identical pressure drop along each inlet. Fig. 1B shows the design of the merged-inlet micromixer with two merged inlets and one outlet, where one merged inlet can supply a mixture of an organic molecule (solute) and solvent, and the other merged inlet includes antisolvent. ESI $\uparrow$ animated movie file shows the internal view of the merged-inlet device. Fig. 1C shows the array of eight such micromixers with 16 merged inlets and 8 outlets. These inlets can be further consolidated using the hydraulic network, as shown in Fig. 1D. The merged inlets of micromixers are connected with three hydraulic networks. The top hydraulic network (blue shaded) allows variation in the solute concentration in each well. The hydraulic network in the bottom half (yellow colored) feeds antisolvent to the micromixers. The flow rates in these networks control the ratio of solvent to antisolvent in each well. The third hydraulic network (green shaded) removes excess crystals (or slurry) from the wells.

\subsection{Computational fluid dynamics simulation of merged-inlet device}

The effectiveness of mixing in the merged-inlet micromixers is evaluated by calculating the velocity, pressure, and concentration profiles in the device. The dimensions of the merged-inlet micromixers and the computer-aided design (CAD) files are provided in the section S1 of the ESI. $\dagger$ The CAD file for design in Fig. 1B was imported in COMSOL Multiphysics ${ }^{\circledR}$ and meshed using a free tetrahedral mesh of element size 0.725 to $3.42 \mathrm{~mm}$ and a curvature factor of 0.9 . The boundary meshes were set at level 2 with a stretching factor of 1.2 and a thickness adjustment factor of 5 . The sharp edges of the corner were trimmed for the corners with angles in the range of $50-240^{\circ}$. The elements were refined using a size scaling factor of 0.35 . Navier Stokes equation coupled with continuity equation was solved for mixing of pure streams of solvent (water) and antisolvent (ethanol). The 
(A) Multi-Inlet Single Well

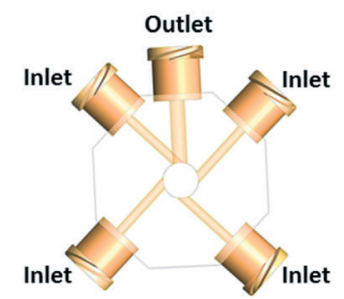

(C) Array of Merged-Inlet Wells

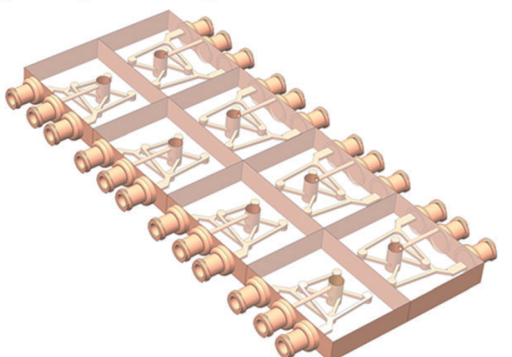

(B) Merged-Inlet Single Well

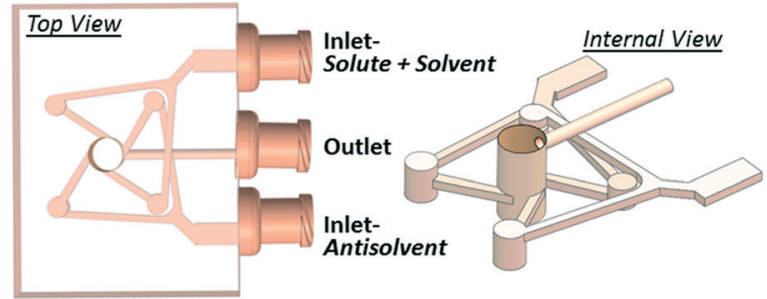

\section{(D) Array of Merged-Inlet Wells with Hydraulic Network}

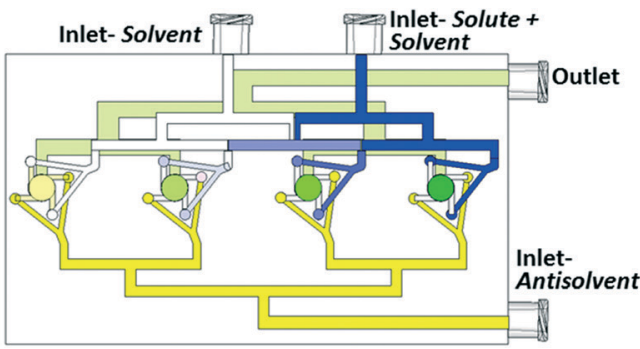

Fig. 1 (A) A conventional multi-inlet micromixer with four inlets tangentially connected to the bottom layer of the cylindrical well and an outlet connected to the top layer of the well. This configuration of inlets and outlet creates cyclonic flow in the cylindrical well for efficient mixing of liquids and for trapping nucleated crystals. (B) Merged-inlet design where alternate (non-neighboring) inlets are merged using a Y-junction such that all the ports are on one side of the device. The internal view shows the vertical offset in the Y-junction of merged inlets to avoid the intersection between channels. (C) An array of eight $(4 \times 2)$ merged-inlet micromixers for parallel screening application. Here, the separate inlets for solvent (8) and antisolvent (8) provides flexibility in changing solvent/antisolvent ratio and solute concentration in each well simultaneously. (D) $4 \times 1$ array of merged-inlet micromixers connected with three hydraulic networks. The top hydraulic network (blue shaded) on the upper half has two inlets coming from the north direction - one for the solution of solute and solvent and the other for pure solvent. The second hydraulic network (yellow colored) on the bottom half has an inlet for antisolvent coming from the east direction. The third hydraulic network (green shaded) located underneath the solvent feed (blue shaded) collects all the slurry from the micromixer and has an outlet towards the east direction.

solute was not considered in the simulation as its solubility is typically at least three orders of magnitude smaller than solvent and antisolvent concentration, which has a negligible effect on the mixing profile. The boundary conditions on inlets were set according to the entering flow rates of water and ethanol. The boundary condition at the outlet was set to zero diffusive flux for continuity equation and fixed ambient pressure for Navier Stokes equation. ( $c f$. section S2 in the ESI $\dagger$ ). The concentration-dependent viscosity and density of the water-ethanol mixture were considered in the model. ${ }^{11}$ The model parameters are given in section S2 of the ESI. $\dagger$ The coupled equations were solved using an iterative solver generalized minimum residual, combined with the algebraic multigrid method. The residual tolerance was set to 0.01 with 200 iterations and left preconditioning. Newton's method was used for iterations with a damping factor of 0.1 and relative tolerance of 0.001 .

\section{Experimental methods}

\subsection{Materials}

The crystalline Form A (stable) of L-histidine w and crystalline form I (stable) of $o$-aminobenzoic acid (o-ABA) (SigmaAldrich, chemical purity $\geq 98 \%$ ) were used for parallel screening study and solubility measurements. The solutions for antisolvent crystallization studies were made using deionized water (Sigma-Aldrich, $18 \mathrm{M} \Omega \mathrm{cm}$ ) and ethanol (Sigma-Aldrich, ACS 99.8\%).

\subsection{Fabrication of microfluidic devices}

The 3D designs of the microfluidic device shown in Fig. 1B and C were designed in SolidWorks ${ }^{\circledR}$ (2018, Dassault Systems) and then 3D printed using a stereolithography (SLA) 3D printer (form 2, Formlabs Inc., USA). A clear FLGPCL02 resin activated by $405 \mathrm{~nm}$ laser was used to $3 \mathrm{D}$ print optically clear microfluidic devices with $150 \mu \mathrm{m}$ of lateral and $25 \mu \mathrm{m}$ of axial resolutions. The clear resin is chemically resistant to various solvents, including ethanol and water. The printed devices were washed with isopropyl alcohol (IPA) (90\%, Sigma-Aldrich) bath for $20 \mathrm{~min}$ in the form wash (Formlabs Inc., USA) to remove the residues of the resin from the external surface. The interior channels of the 3D printed device were washed separately by injecting IPA using a syringe. The post-washed 3D printed devices were finished by removing supports and then curing for 20 min using a commercial ultraviolet lamp. The optical transparency of the 3D printed microfluidic device was improved by wet sanding using 400 to 12000 grit pads followed by spray painting of resin. The top and bottom openings of the micromixers in the multi-well device (see Fig. 2B) were sealed with polycarbonate films for optical clarity to image crystals under the microscope. The fabricated multi-well device is shown in Fig. 2A and B. 


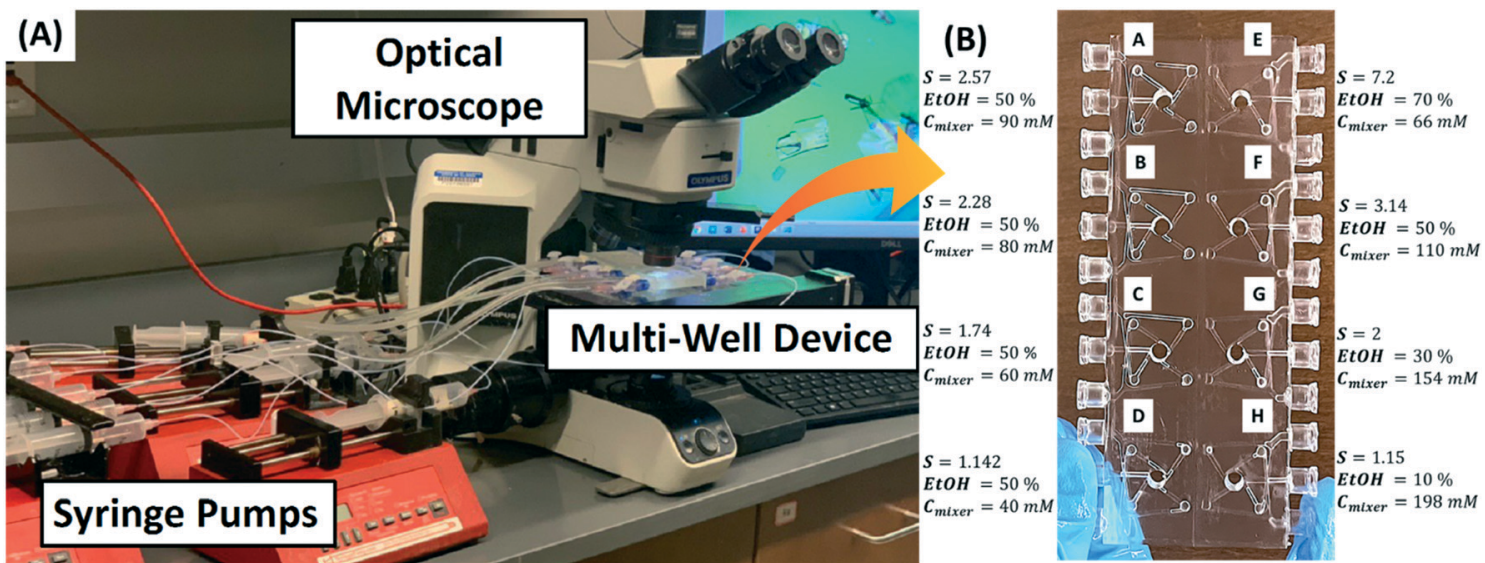

Fig. 2 (A) Experimental setup for parallel screening. It involves syringe pumps pushing aqueous solution and antisolvent at a constant flow rate to in a multi-well microfluidic device, which is continuously monitored under the optical microscope. (B) Picture of a 3D printed multi-well device with wells labeled from $A$ to $H$. The supersaturations in wells $A$ to $D$ were controlled by varying L-histidine concentration, and supersaturations in wells $\mathrm{E}$ to $\mathrm{H}$ were controlled by varying vol\% of ethanol.

\subsection{Experimental setup and operation of multi-well device}

Fig. 2A shows the experimental setup for parallel screening, which involves flow crystallization in a multi-well microfluidic device that is continuously monitored under the optical microscope (Olympus BX53M, Olympus America Inc.). This setup was first benchmarked to reproduce previously reported screening results for $o$-ABA, ${ }^{1}$ and then applied to screen morphology, polymorph, and growth rates of L-histidine for different solvent ratios, solute concentrations, and supersaturations. L-histidine was grown in a multi-well device using the antisolvent method implemented by mixing an aqueous solution of L-histidine with pure ethanol (antisolvent) at room temperature $\left(\sim 20{ }^{\circ} \mathrm{C}\right)$. The aqueous solution of L-histidine and pure ethanol were pumped separately into each microwell in the multi-well device using programmed syringe pumps (NE-4000, New Era Pump System Inc.). The inlets are connected to one-way microfluidic check valves to prevent backflow inside the channels. The solubility of L-histidine in the ethanol-water mixture was measured in the single merged-inlet device according to the procedure described in section 3.4. To achieve desired supersaturation in the micromixer, the required concentration of L-histidine and the volume fraction of ethanol in the ethanol-water mixture were determined from the solubility curve, which was controlled by mixing streams of a concentrated aqueous solution of L-histidine and pure ethanol. For instance, a supersaturation of 2 corresponding to the solubility limit of $0.078 \mathrm{~mol} \mathrm{~L}^{-1}$ in $30 \mathrm{vol} \%$ ethanol can be achieved by feeding an aqueous solution of $0.22 \mathrm{~mol} \mathrm{~L}^{-1}$ of L-histidine at $0.7 \mathrm{ml}$ $\min ^{-1}$ and $100 \%$ ethanol at $0.3 \mathrm{ml} \mathrm{min}^{-1}$ to the micromixer. Here, the vol\% is calculated based the volume of pure ethanol and water mixed at room temperature $\left(\sim 20{ }^{\circ} \mathrm{C}\right)$. Fig. 2B shows the supersaturation in each well of the multiwell device labeled A to $\mathrm{H}$. The supersaturations in wells A to $\mathrm{D}$ were controlled by varying concentrations of L-histidine in water fed while feeding ethanol at equal flow rates of $0.5 \mathrm{ml}$ $\min ^{-1}$, whereas the supersaturations in wells $\mathrm{E}$ to $\mathrm{H}$ were varied for fixed L-histidine concentration in water mixed with different flow rates of ethanol to achieve different vol\% of ethanol in the mixer. The total flow rate of aqueous solution and ethanol was maintained at $1 \mathrm{ml} \mathrm{min}^{-1}$ in all the wells to have similar residence time of supersaturated solution in the micromixers. The composition and flow rates of each stream entering the wells A to $\mathrm{H}$ are provided in the section $\mathrm{S} 3$ of the ESI. $\dagger$ All the conditions in eight wells A-H were repeated thrice to obtain the error bars.

The effective startup of a multi-well device is crucial for the consistent screening of crystallization. The multi-well device was first flushed with the aqueous solution of L-histidine to remove air from the channels and micromixers, and then ethanol was injected at a sufficiently higher total flow rate to attain steady concentration and flow profiles in a time duration much smaller than the induction time of the crystals. The induction time of L-histidine decreases with increasing vol\% of ethanol and supersaturation with the longest time reported of $\sim 40 \mathrm{s.}^{12}$ Therefore, the startup time for this study was less than a few seconds. Since the startup time is close to the average residence time (see residence time distribution in ESI $\dagger$ ) of the micromixer that has a volume of $0.8 \mathrm{ml}$, the initial total flow rate was set to $5 \mathrm{ml}$ $\min ^{-1}$ for $10 \mathrm{~s}$ followed by a steady flow rate of $1 \mathrm{ml} \mathrm{min} \mathrm{m}^{-1}$ for $30 \mathrm{~min}$. The microscopic images were recorded with a built-in color camera (LC 30, Olympus America Inc.). The time-lapsed images were captured consecutively at an interval of 2 min on each well during the total crystallization time of $30 \mathrm{~min}$.

\subsection{Solubility estimation}

The solubility data for $o$-ABA in the water-ethanol mixture was obtained from the literature. ${ }^{1,13}$ The solubility of L-histidine in ethanol-water mixtures was measured at room temperature $\left(20^{\circ} \mathrm{C}\right)$. The solubility measurements were 
conducted in the multi-inlet single well device (Fig. 1A) by manipulating flow rates of four inlets, labeled as 1: pure ethanol, 2: pure water, 3 and 4: joint inlet for a saturated solution of L-histidine in water, to observe nucleated crystals in a specific time interval. For example, the solubility at 50 vol\% ethanol was measured by setting the flow rate of inlet-1 to $0.5 \mathrm{ml} \mathrm{min} \mathrm{min}^{-1}$, and initial flow rates of inlet-2 to $0.5 \mathrm{ml}$ $\min ^{-1}$ and joint inlet-3 and 4 to $0 \mathrm{ml} \min ^{-1}$. While keeping the total flow rate of inlet-2, and joint inlet-3 and 4 to $0.5 \mathrm{ml}$ $\min ^{-1}$, the flow rate of a saturated aqueous solution of L-histidine in the joint inlet was increased in a stepwise manner until nucleated crystals are observed in 1 min time step. The concentration of L-histidine in the micromixer, calculated based on the flow rates of all inlets leading to the nucleation event, provides the solubility at a fixed ethanol vol\%. Such a dynamic measurement of solubility is sensitive to the time interval for observation of nucleation during a stepwise increase in the flow rate of the joint inlet. Fig. S4 in the ESI $\dagger$ shows the measured values of solubility of L-histidine using this dynamic technique for $1,3,5$, and 10 min of interval for observation of nucleation. The observed (or apparent) solubility decreases with increasing this time
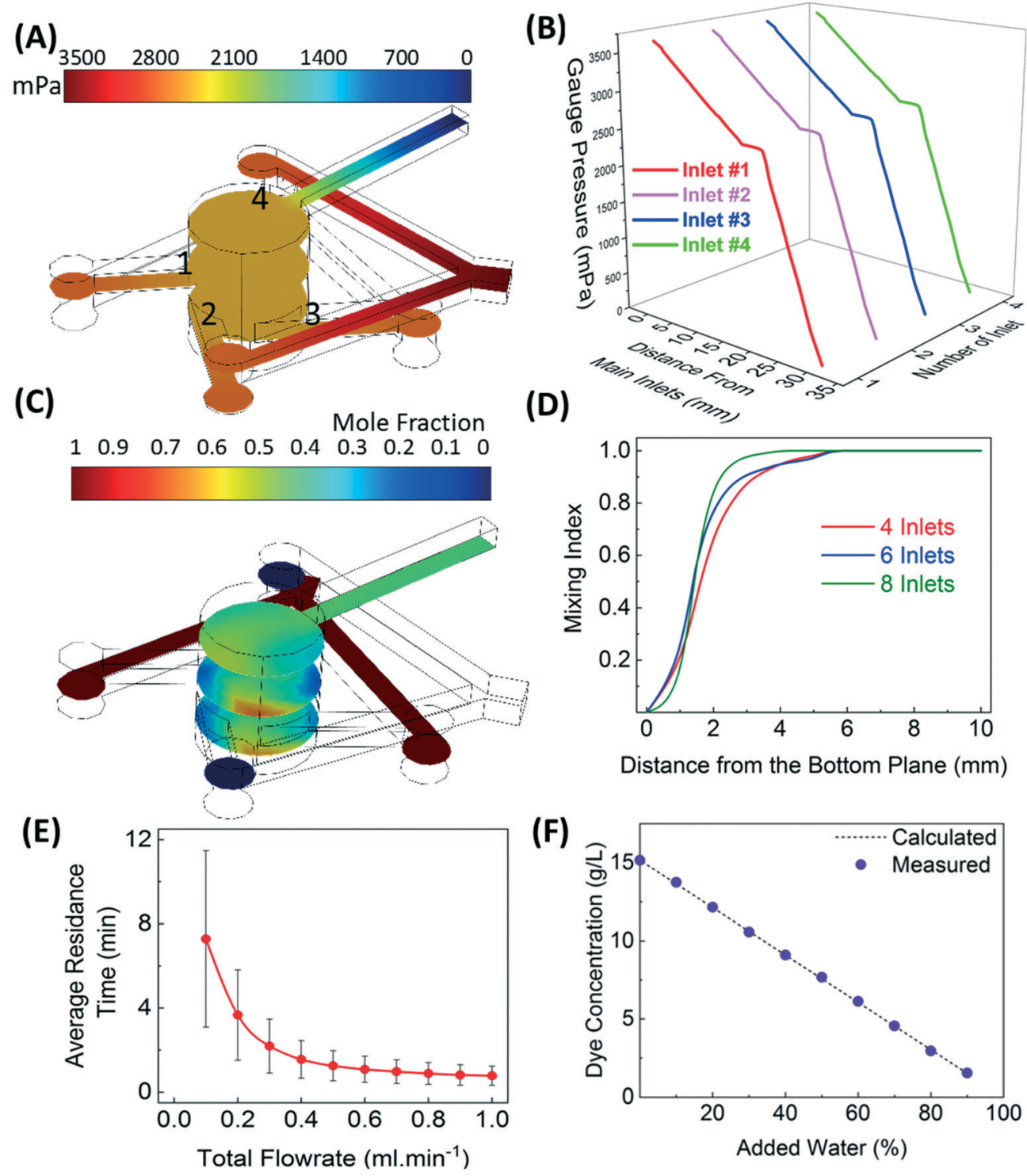

Distance from the Bottom Plane $(\mathrm{mm})$

(F)

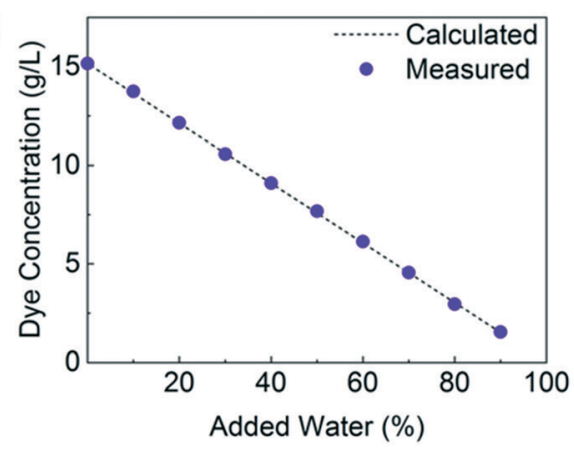

Fig. 3 (A) Variation in the gauge pressure of ethanol-water mixture flowing upward in the micromixer for the inlet flow rate of $0.5 \mathrm{ml}^{\mathrm{min}}{ }^{-1}$ for ethanol and $0.5 \mathrm{ml} \mathrm{min} \mathrm{m}^{-1}$ for water. (B) Decrease in the gauge pressure along the axial direction of a channel for flow condition in (A). The pressure drop along each inlet is identical. (C) Variation in the mole fraction of ethanol in the mixer for the inlet flow rate of $0.5 \mathrm{ml} \mathrm{min}{ }^{-1}$ of ethanol and 0.5

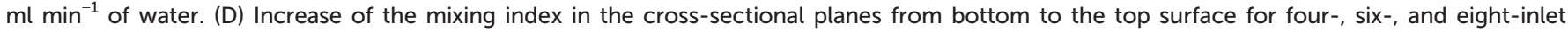
micromixers. Almost homogeneous mixing is observed at a height greater than $1.8 \mathrm{~mm}$. (E) Average residence time distribution and variance of the micromixer as a function of flow rate. (F) Verification of homogeneous mixing from dye experiment, where $(1-x) \mathrm{ml} \mathrm{min}^{-1}$ of $15 \mathrm{~g} \mathrm{l}^{-1}$ of aqueous dye solution is mixed with $x \mathrm{ml} \mathrm{min}{ }^{-1}$ of water. Dilution of dye at the outlet with an increasing volume fraction of added water $(x)$ matches well with the calculated values, which confirms homogeneous mixing with no dead volume in the micromixer. 
interval, where the solubility data obtained for $10 \mathrm{~min}$ interval matches well with the thermodynamic solubility. ${ }^{14,15}$ The variation in solubility from $1 \mathrm{~min}$ to $10 \mathrm{~min}$ observation interval can be interpreted as metastable zone width for nucleation. A more accurate estimation of solubility can be made by measuring solution composition for zero growth condition. ${ }^{16}$

\subsection{Measurement of growth rates, morphology, and polymorphs}

The time-lapsed images were processed to measure the size and shape distribution of crystals using image analysis in Olympus Stream Start. Details of image processing and analysis can be found in ref. 17. The polymorphic forms of $o$-ABA and L-histidine were distinguished based on their distinct morphological forms. For several organic crystals, there are unique morphologies associated with different polymorphic forms that can be identified using software Morphology Domain. ${ }^{18,19}$ Form I of $o$-ABA has prismatic morphology, whereas form II takes needle-like morphology. ${ }^{1}$ The form A (stable) of L-histidine has rod-like morphology, and form B (metastable) has plate-like morphology with dominant (100), (110), and (111) faces (see section S6 of the ESI $\dagger$ ). ${ }^{14,15,20,21}$ The rod-like crystals of form $A$ were distinguished from the plate-like crystals of form B based on applying a cutoff of $>2$ on the aspect ratio in the image analysis program. The percentage of form A was calculated based on the fraction of the area covered by the rod-like crystals. The polymorphic forms of L-histidine were also confirmed from X-ray diffraction (XRD) experiments on a Bruker D2 PHASER diffractometer using Ni filtered $\mathrm{Cu} \mathrm{K \alpha}$ radiation. For all samples, a step width $2 \theta$ of $0.2^{\circ}$ and a counting time of $5 \mathrm{~S}$ per step were used to enhance the signal-to-noise ratio. XRD patterns of samples of L-histidine are provided in section $\mathrm{S} 7$ of ESI. $\dagger$

\section{Results and discussion}

\subsection{Pressure variation and mixing in the merged-inlet device}

The alternate inlets of the merged-inlet device are combined using a Y-junction to ensure an even split of flow and uniform pressure inside the micromixer. Fig. 3A shows a decrease in the gauge pressure of ethanol flowing at $0.5 \mathrm{ml}$ $\min ^{-1}$ in inlets 1 and 3 and water flowing at $0.5 \mathrm{ml} \mathrm{min}^{-1}$ in inlets 2 and 4, which are entering into the mixer and leaving from the outlet of the device. The pressure change in the micromixer is within a few pascals and uniform across the cross-sectional planes. Fig. 3B shows the gauge pressure drop along the axial direction starting from different inlets emerging from the Y-junction to the outlet. The pressure drop is identical in non-neighboring inlets, which confirms identical flow rates in each pair of merged inlets.

The concentration distribution of ethanol and water as they are combined in the mixer at identical flow rates of 0.5

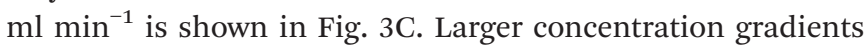
are observed near the inlets as compared to the central zone. These gradients are inevitable but can be reduced by increasing the number of inlets and the flow rates. ${ }^{1}$ Fig. 3D shows an increase in the mixing index of a cross-sectional plane of the mixer with increasing the distance from the bottom plane for 4-, 6-, and 8-inlet mixers. The mixing index is defined as $\left(1-\sigma^{2} / \sigma_{0}^{2}\right)$, where $\sigma^{2}$ is the variance of concentration in any cross-sectional plane and $\sigma_{0}^{2}$ is the variance in concentration of the bottom plane of the mixer. The dynamic change in the mixing can be interpreted from
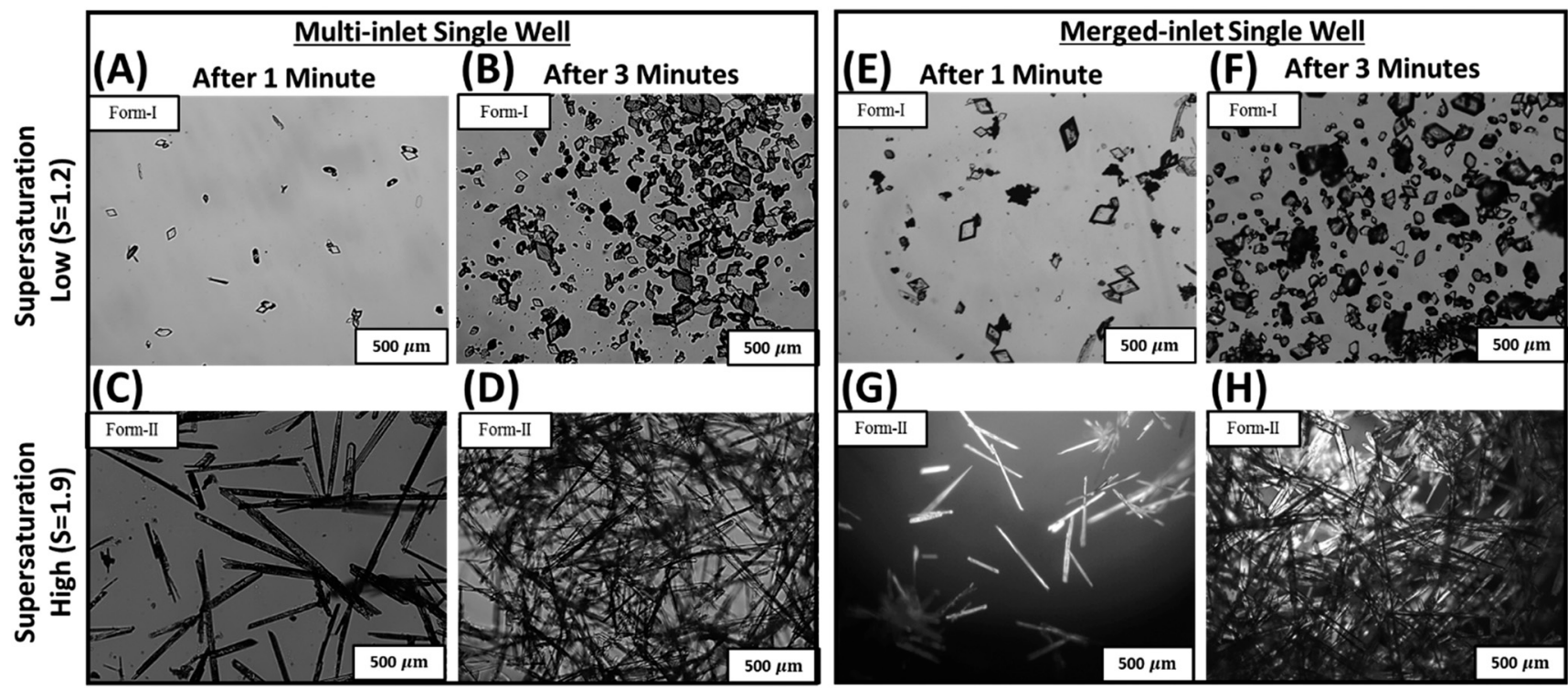

Fig. 4 Comparison of morphologies and polymorphs of o-ABA at two different supersaturations in the multi-inlet device (A-D) and merged-inlet device $(E-H)$. Both multi-inlet and merged-inlet devices show form-I polymorph of prismatic morphology at supersaturation 1.2 (A and B, vs. $E$ and F), and form-Il polymorph of needle-like morphology at supersaturation 1.9 (C and D, vs. G and H). 
the residence time distribution (RTD) of the mixer. Fig. 3E shows the decrease in the average residence time and the standard deviation of RTD with the increasing total flow rate of the fluid. The dye experiments were also conducted to confirm efficient mixing of $(1-x) \mathrm{ml} \mathrm{min}^{-1}$ of $15 \mathrm{~g} \mathrm{l}^{-1}$ of

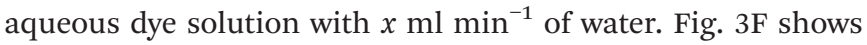
the concentration of diluted dye solution at the outlet of the merged-inlet device measured at $300 \mathrm{~s}$ for a varying percentage of added water.

\subsection{Comparative screening of morphology and polymorphs of} o-ABA in multi-inlet versus merged-inlet device

The performance of the merged-inlet device is first benchmarked by reproducing reported data on morphology and polymorph screening of $o$-ABA. ${ }^{1}$ Fig. $4 \mathrm{~A}-\mathrm{D}$ show two different polymorphs of $o$-ABA - form-I at supersaturation 1.2 and form-II at supersaturation 1.9 - obtained using the multi-inlet device (Fig. 1A). ${ }^{1}$ The ability of micromixer to trap crystals and grow them under continuous feed allows consistent screening of metastable form-II, which is not feasible in microtiter plates. ${ }^{1}$ Fig. $4 \mathrm{E}-\mathrm{H}$ show similar results with the merged-inlet device, where form-I and form-II are identified at supersaturations 1.2 and 1.9, respectively. The reproducibility of morphology and polymorphs in the merged-inlet device confirms its suitability for parallel screening applications using a multi-well device.

\subsection{Parallel screening of morphology, polymorphs, and} growth rate of L-histidine using multi-well device and 96-well microtiter plate

The efficacy of the eight-well device (shown in Fig. 2B) is evaluated here for parallel screening of morphology, polymorph, and growth rates of L-histidine via antisolvent crystallization. The concentration of L-histidine and vol\% of ethanol in each well of the multi-well device are flow controlled to achieve different supersaturations. The composition in each well are labelled as A: $\sigma=2.57,50 \mathrm{vol} \%$ ethanol, B: $\sigma=2.28,50 \mathrm{vol} \%$ ethanol, C: $\sigma=1.74,50 \mathrm{vol} \%$ ethanol, D: $\sigma=1.14,50$ vol\% ethanol, E: $\sigma=7.2,70$ vol\% ethanol, F: $\sigma=3.14,50 \mathrm{vol} \%$ ethanol, G: $\sigma=2.00,30 \mathrm{vol} \%$ ethanol, and $\mathbf{H}: \sigma=1.15,10$ vol\% ethanol. The supersaturation in wells decreases in the order $\mathbf{E}>\mathbf{F}>\mathbf{A}>$ $\mathbf{B}>\mathbf{G}>\mathbf{C}>\mathbf{H} \approx$ D. Fig. 5 A-1, B-1, E-1 and F-1 show dominant plate-like morphology characteristic of form-B (metastable) of L-histidine, whereas the rest other wells C-1, D-1, G-1, and H-1 show a few crystals of rod-like morphology of form-A (stable) after $5 \mathrm{~min}$ in the multi-well device. Fig. A-2 to H-2 show a much large number of crystals with similar morphological and polymorphic characteristics as compared to A-1 to $\mathrm{H}-1$ after $30 \mathrm{~min}$. The rod-like crystals of form-A are more evident in $\mathrm{C}-2, \mathrm{D}-2, \mathrm{G}-2$, and $\mathrm{H}-2$. The polymorphic forms of plate-like and rod-like crystals are also confirmed using XRD shown in the section S7 of the ESI. $\dagger$

The parallel screening performance of multi-well device is compared with a 96-well microtiter plate. The right panels in

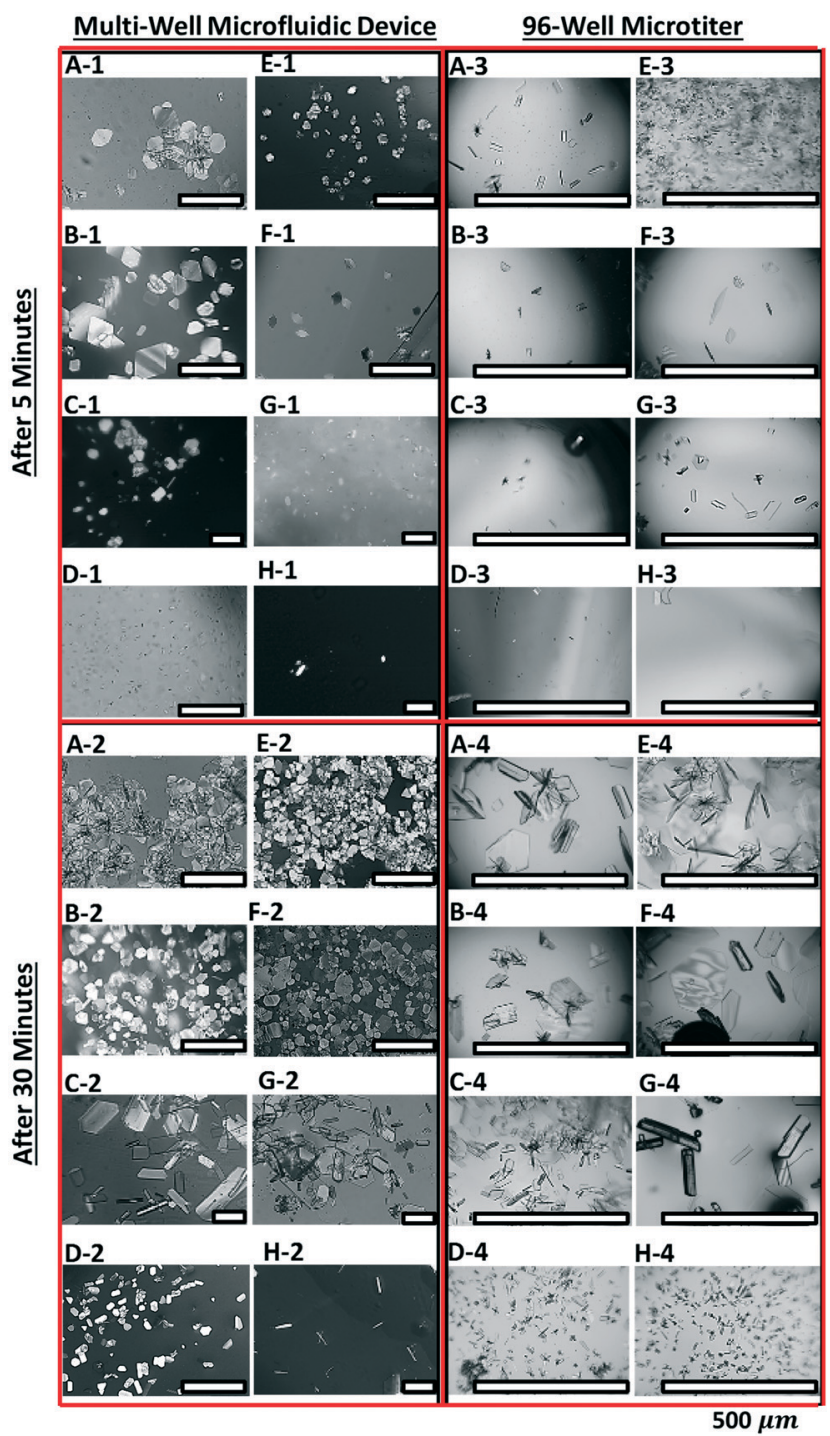

Fig. 5 Screening of crystal morphologies and polymorphs of L-histidine crystals using the multi-well microfluidic device, and the comparison of results with 96-well microtiter plate. Four panels of eight optical micrographs are arranged to compare the micrographs in the left panels (A-1 to H-1 (5 min), and A-2 to H-2 (30 min)) for the multi-well device with the micrographs in the right panels $(A-3$ to $\mathrm{H}-3$ (5 $\mathrm{min})$, and $\mathrm{A}-4$ to $\mathrm{H}-4$ (30 $\mathrm{min})$ ) for 96 -well microtiter. The solution compositions for eight micrographs in each panel corresponds to the supersaturations $(\sigma)$ and vol\% ethanol shown in Fig. 2B, such as A: $\sigma=$ $2.57,50$ vol\% ethanol, B: $\sigma=2.28,50$ vol\% ethanol, C: $\sigma=1.74,50$ vol\% ethanol, D: $\sigma=1.14,50$ vol\% ethanol, E: $\sigma=7.2,70$ vol\% ethanol, F: $\sigma=3.14,50$ vol\% ethanol, G: $\sigma=2.00,30$ vol\% ethanol, and $\mathbf{H}: \sigma=$ $1.15,10$ vol\% ethanol.

Fig. 5 A-3 to H-4 show optical micrographs of crystals grown under identical conditions in $4 \times 2$ array of 96-well device. Very few crystals can be seen in the first 5 min in wells A-3 to $\mathrm{H}-3$, and the majority of these are rod-like crystals. After 30 min, larger crystals of mixed morphology and polymorphs are observed in wells A-4, B-4, E-4, and F-4, whereas the lowsupersaturation wells C-4, D-4, G-4, and H-4 showed dominant, stable form-A. Since the supersaturation in microtiter plates decreases as the crystals grow, a larger 
(A)

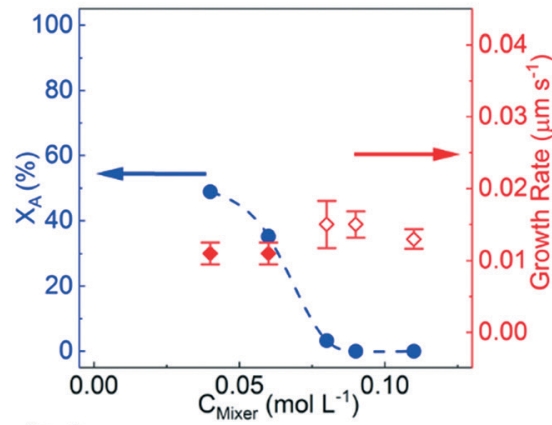

(D)

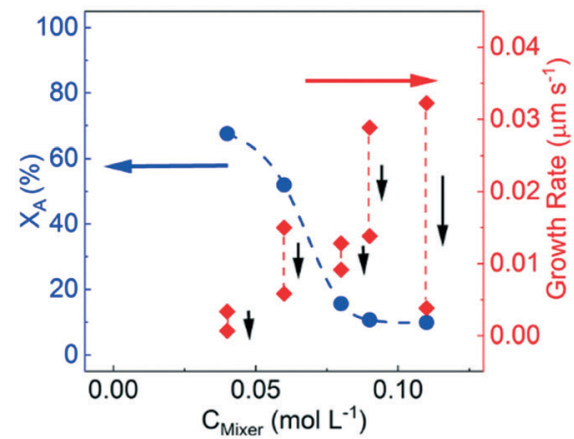

(B)

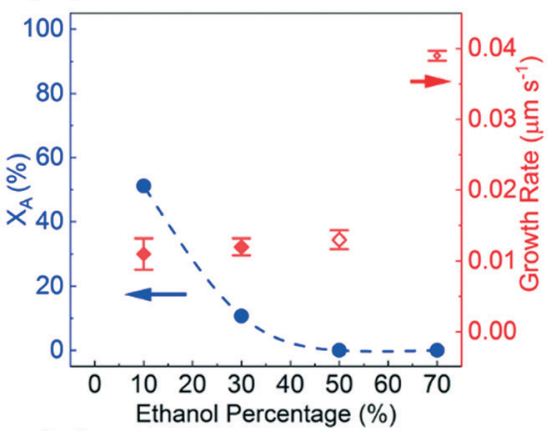

(E)

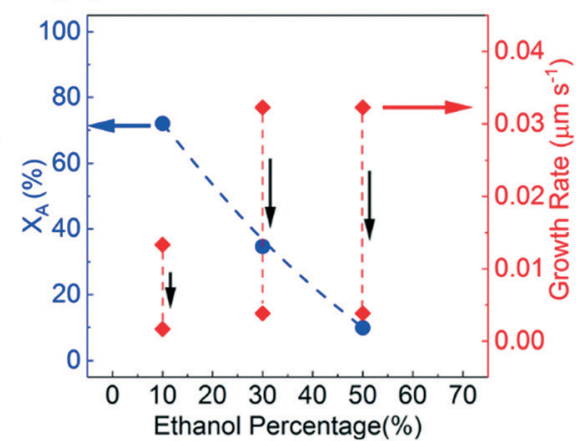

(C)

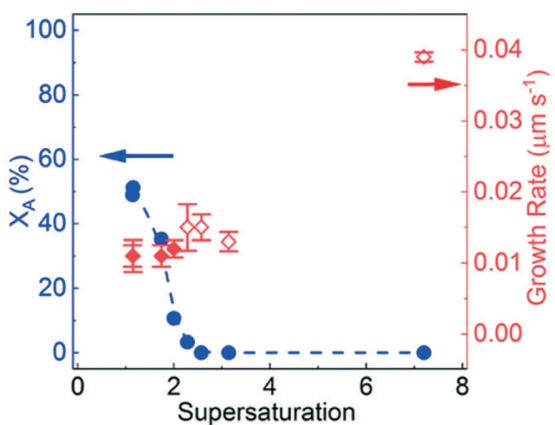

(F)

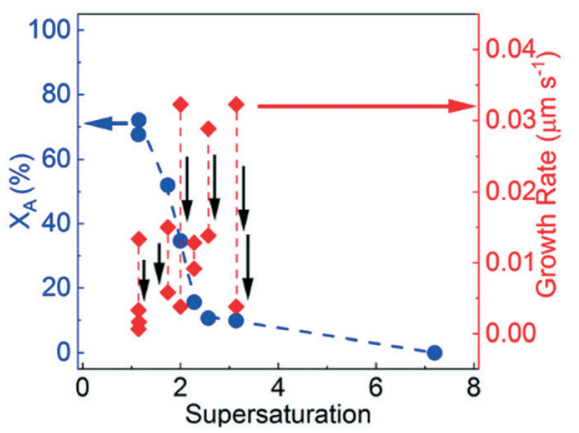

Fig. 6 Variation in the percentage of form-A (solid blue circles) and growth rates of (111) facet of form-A (solid red diamonds) and form-B (open red diamonds) of L-histidine with increasing (A) concentration of L-histidine $\left(C_{\text {Mixer }}\right)$ in the multi-well device, (B) volume percentage of ethanol in the multi-well device, (C) supersaturation in the multi-well device, (D) concentration of L-histidine in the 96-well device, (E) volume percentage of ethanol in the 96-well device, and (F) supersaturation in the 96-well device. The black arrows in (D)-(F) represent a decrease in the growth rate in $30 \mathrm{~min}$ due to depletion of supersaturation in the 96-well device. The red and blue arrows indicate $y$-axes for the growth rate and percentage of the stable form, respectively.

fraction of metastable crystals can transform into stable crystals via Ostwald ripening. This is the primary reason for the disappearance of plate-like crystals (metastable) in wells A-4, B-4, E-4, and F-4.

The percentage of stable form- $\mathrm{A}\left(X_{\mathrm{A}}\right)$ and the growth rates of (111) facet for different compositions in each well are obtained from image analysis (as described in section 3.5). Fig. 6A-C show the variation in $X_{\mathrm{A}}$ and growth rates as a function of L-histidine concentration, vol\% of ethanol, and supersaturation, respectively, for a multi-well device. Fig. 6A shows the percentage of $X_{\mathrm{A}}$ decreases with increasing concentration of L-histidine in the mixer at fixed $50 \mathrm{vol} \%$ of ethanol, and it becomes negligible for concentration $>75 \mathrm{mM}$. However, the growth rate increases marginally with L-histidine concentration. Fig. 6B shows a decrease in the percentage of $X_{\mathrm{A}}$ with increasing $\mathrm{vol} \%$ of ethanol at a fixed L-histidine concentration of $220 \mathrm{mM}$ in the feed. The percentage of $X_{\mathrm{A}}$ is almost zero for $>50$ vol\% of ethanol. The combined effect of L-histidine concentration and vol\% of ethanol can be represented as supersaturation, as shown in Fig. 6C. While the percentage of form-A drops sharply from supersaturation 1 to 2 , the growth rate increases linearly from 0.010 to $0.015 \mu \mathrm{m} \mathrm{s}^{-1}$. The metastable form-B dominantly occurs for supersaturation $>2.2$, with growth rate increasing exponentially from $0.017 \mu \mathrm{m} \mathrm{s}^{-1}$ at supersaturation 2.2 to 0.04 $\mu \mathrm{m} \mathrm{s}^{-1}$ at supersaturation 7 .
Fig. $6 \mathrm{D}-\mathrm{F}$ show the variation in $X_{\mathrm{A}}$ and growth rates as a function of L-histidine concentration, vol\% of ethanol, and supersaturation, respectively, in 96-well microtiter device. Although the microtiter plate qualitatively captures the trend in the percentage of $X_{\mathrm{A}}$ for varying concentrations, vol\% of ethanol, and supersaturations, it overestimates the percentage of stable form $\left(X_{\mathrm{A}}\right)$. The overestimation of stable form is due to dissolution of metastable form that occurs when supersaturation decreases below the solubility of the metastable form. The depletion of supersaturation in microtiter plates greatly impacts the growth rate measurement, which constantly decreases over time and cannot be reliable.

\section{Conclusion}

An effective design of a continuous-flow, well-mixed, multiwell microfluidic device is presented here for parallel screening of crystalline materials at controlled conditions. The multiple tangential inlets of the micromixer are consolidated to make an array of wells without disrupting the cyclonic/vortex flow pattern inside the micromixers. A merged-inlet configuration utilizing Y-junctions effectively reduces the 32 inlets (four tangential inlets to each micromixer) in an array of eight micromixers to 16 inlets, 
which can be further reduced to three inlets using the hydraulic network. The reduction of the total number of inlets reduces the number of pumps required for parallel screening using such a multi-well device.

The identical pressure drops in non-neighboring, alternate inlets confirm uniform flow rates in each inlet entering the micromixer. The negligible pressure drop in the micromixer and higher mixing index ensures homogeneous mixing of entering fluid, which is required to achieve homogeneous supersaturation rapidly in the micromixer. The average residence time of fluid can be controlled by varying the flow rates so that the startup time is much higher than the induction time of crystal nucleation. This is an important requirement to obtain reliable and reproducible data from such flow studies.

The impact of this multi-well device stems from its ability to conduct parallel screening and reduce the time required to evaluate multiple crystallization conditions. While the time required for sequential screening using a single well device increases linearly with a number of conditions, the parallel screening using a multi-well device can substantially reduce overall screening time. Here, we demonstrate screening of crystal morphology, polymorph, and growth rates of L-histidine for eight different conditions such as molar concentration, vol\% of ethanol, and supersaturation in $\sim 30$ min. In contrast, the sequential screening would take $8 \times 30$ $=240 \mathrm{~min}$ to conduct a similar study. The effectiveness of the multi-well device is also compared with the traditional 96well microtiter device to evaluate the impact of varying supersaturation in microtiter plates on screening results. In general, the microtiter plates overestimate the percentage of stable polymorph as compared to metastable polymorph with large variations in the growth rates. At the same time, the continuous-flow multi-well device provides reliable and robust quantification of crystal polymorphs, morphology, and growth rates.

This design will eventually lead to the development of high-throughput material screening devices that can trap varieties of crystalline materials and study them under controlled conditions.

\section{Disclosure}

Data were generated by the University of Illinois at Chicago. AbbVie Inc., North Chicago, USA, provided experimental support for a summer internship of Paria Coliaie, Dr. Manish Kelkar, and Dr. Nandkishor K. Nere are present employees of AbbVie Inc.

\section{Conflicts of interest}

A PCT application (PCT/US20/36353) titled "Continuous-Flow, Well Mixed, Microfluidic Crystallization Device for Screening Polymorphs, Morphologies and Crystallization Kinetics at Controlled Supersaturation," has been filed.

\section{Acknowledgements}

This material is based on the work performed in the Materials and Systems Engineering Laboratory at the University of Illinois at Chicago in collaboration with Enabling Technology Consortium (ETC: https://www.etconsortium.org/, specifically Abbvie, Biogen, Bristol Myers Squibb, and Takeda Pharmaceuticals). P. C. and M. R. S. acknowledge funding support from ETC to conduct this work. P. C. acknowledges the summer internship opportunity at AbbVie Inc. to conduct a few experiments for the completion of this work. The authors also thank Moussa Boukerche, Jie Chen, Daniel Pohlman, Bradley Greiner, Pankaj Shah, and Kushal Sinha for their comments and suggestions. The authors would also like to acknowledge the leadership support from Samrat Mukherjee, Ahmad Sheikh, and Shailendra Bordawekar of AbbVie Inc.

\section{References}

1 P. Coliaie, M. S. Kelkar, N. K. Nere and M. R. Singh, Continuous-flow, well-mixed, microfluidic crystallization device for screening of polymorphs, morphology, and crystallization kinetics at controlled supersaturation, $L a b$ Chip, 2019, 19, 2373-2382.

2 J. Puigmartí-Luis, Microfluidic platforms: a mainstream technology for the preparation of crystals, Chem. Soc. Rev., 2014, 43, 2253-2271.

3 Q. Zhao, H. Cui, Y. Wang and X. Du, Microfluidic platforms toward rational material fabrication for biomedical applications, Small, 2020, 16, 1903798.

4 P. M. Valencia, O. C. Farokhzad, R. Karnik and R. Langer, Microfluidic technologies for accelerating the clinical translation of nanoparticles, Nat. Nanotechnol., 2012, 7, 623-629.

5 J.-L. Fraikin, T. Teesalu, C. M. McKenney, E. Ruoslahti and A. N. Cleland, A high-throughput label-free nanoparticle analyser, Nat. Nanotechnol., 2011, 6, 308-313.

6 H. Wang, K. Liu, K.-J. Chen, Y. Lu, S. Wang, W.-Y. Lin, F. Guo, K.-I. Kamei, Y.-C. Chen and M. Ohashi, A rapid pathway toward a superb gene delivery system: programming structural and functional diversity into a supramolecular nanoparticle library, ACS Nano, 2010, 4, 6235-6243.

7 Y. Kim, B. Lee Chung, M. Ma, W. J. Mulder, Z. A. Fayad, O. C. Farokhzad and R. Langer, Mass production and size control of lipid-polymer hybrid nanoparticles through controlled microvortices, Nano Lett., 2012, 12, 3587-3591.

8 M. D. Brennan, M. L. Rexius-Hall, L. J. Elgass and D. T. Eddington, Oxygen control with microfluidics, Lab Chip, 2014, 14, 4305-4318.

9 C. W. Shields IV, C. D. Reyes and G. P. López, Microfluidic cell sorting: a review of the advances in the separation of cells from debulking to rare cell isolation, Lab Chip, 2015, 15, 1230-1249. 
10 S. K. Dertinger, D. T. Chiu, N. L. Jeon and G. M. Whitesides, Generation of gradients having complex shapes using microfluidic networks, Anal. Chem., 2001, 73, 1240-1246.

11 C. Galletti, G. Arcolini, E. Brunazzi and R. Mauri, Mixing of binary fluids with composition-dependent viscosity in a T-shaped micro-device, Chem. Eng. Sci., 2015, 123, 300-310.

12 C. M. Roelands, S. Jiang, M. Kitamura, J. H. ter Horst, H. J. Kramer and P. J. Jansens, Antisolvent crystallization of the polymorphs of L-histidine as a function of supersaturation ratio and of solvent composition, Cryst. Growth Des., 2006, 6, 955-963.

13 S. Jiang, J. H. ter Horst and P. J. Jansens, Concomitant polymorphism of o-aminobenzoic acid in antisolvent crystallization, Cryst. Growth Des., 2007, 8, 37-43.

14 L. Wantha, N. Punmalee, V. Sawaddiphol and A. E. Flood, Effect of ethanol on crystallization of the polymorphs of $\mathrm{L}^{-}$ histidine, J. Cryst. Growth, 2018, 490, 65-70.

15 M. Kitamura, Crystallization behavior and transformation kinetics of L-histidine polymorphs, J. Chem. Eng. Jpn., 1993, 26, 303-307.

16 M. R. Singh, N. Nere, H.-H. Tung, S. Mukherjee, S. Bordawekar and D. Ramkrishna, Measurement of Polar Plots of Crystal Dissolution Rates Using Hot-Stage Microscopy. Some Further Insights into Dissolution Morphologies, Cryst. Growth Des., 2014, 14, 5647-5661.

17 M. R. Singh, J. Chakraborty, N. Nere, H. H. Tung, S. Bordawekar and D. Ramkrishna, Image-Analysis-Based Method for 3D Crystal Morphology Measurement and Polymorph Identification Using Confocal Microscopy, Cryst. Growth Des., 2012, 12, 3735-3748.

18 M. R. Singh, P. Verma, H.-H. Tung, S. Bordawekar and D. Ramkrishna, Screening Crystal Morphologies from Crystal Structure, Cryst. Growth Des., 2013, 13, 1390-1396.

19 M. R. Singh and D. Ramkrishna, A Comprehensive Approach to Predicting Crystal Morphology Distributions with Population Balances, Cryst. Growth Des., 2013, 13, 1397-1411.

20 L. Wantha, N. Punmalee and A. E. Flood, Influence of Solvents on Solution-Mediated Polymorphic Transformation of the Polymorphs of L-Histidine, Chem. Eng. Technol., 2019, 42, 1505-1511.

21 L. Wantha and A. E. Flood, Growth and Dissolution Kinetics of A and B Polymorphs of L-Histidine, Chem. Eng. Technol., 2015, 38, 1022-1028. 